\title{
CANCER
}

\section{Microtubule-straightening compound widens the therapeutic window}

this MTA
causes subtle
changes in
the curvature
between
aß-tubulin
heterodimers
that coincide
with
dramatically
altered
microtubule
dynamics

Microtubule-targeting agents (MTAs) are standard-of care chemotherapeutics for a wide range of cancers, but their use is limited by side effects such as neuropathy. Now, reporting in Science Translational Medicine, Brouhard, Roulston and colleagues present a novel small-molecule MTA that has potent antitumour activity in several different cancer models, but does not induce neurotoxicity at effective doses.

Microtubules are components of the cytoskeleton, and as such, have a central role in many cellular processes. During cell division, microtubules are major constituents of mitotic spindles, which makes them attractive targets for cancer therapy. They consist of $\alpha \beta$-tubulin heterodimers that are polymerized into protofilaments, which then associate laterally to form a tube. Growth occurs through the addition of $\alpha \beta$-tubulin dimers to the

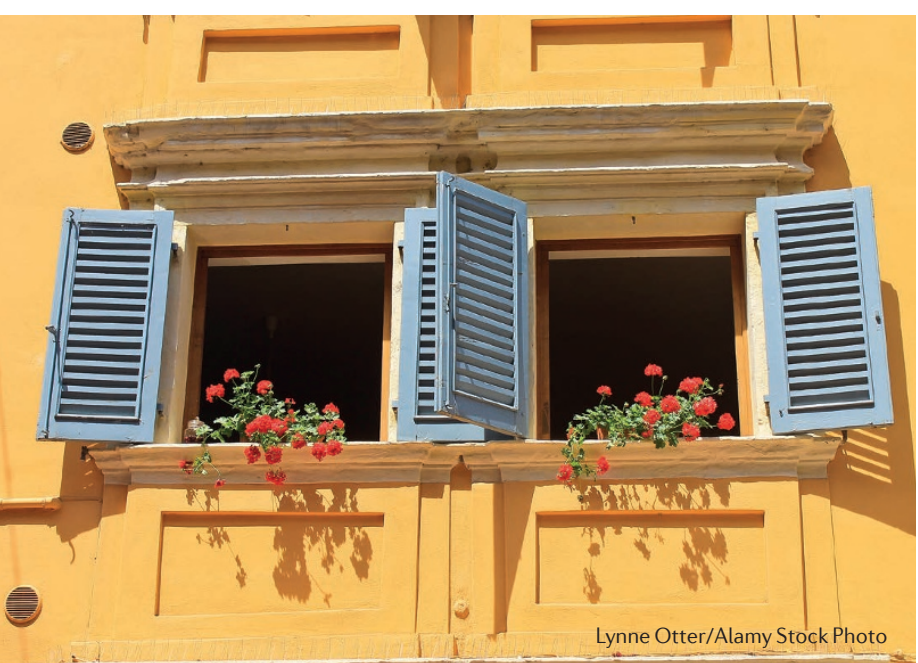

microtubule ends, whereas shrinkage is associated with 'fraying' of the protofilaments, which bend outwards and depolymerize. The switch from growth to shrinkage is termed catastrophe; however, sporadic 'rescue' events can revert the microtubule to growth mode.

MTAs are known to alter the dynamics and stability of microtubules, and this leads to an arrest in mitosis in dividing cells, which ultimately triggers apoptosis. However, MTAs also affect non-dividing cells, in particular neurons, as the backbone of neuronal axons consists of microtubules. Investigating a compound called DZ-2384, the authors found that this MTA causes subtle changes in the curvature between $\alpha \beta$-tubulin heterodimers that coincide with dramatically altered microtubule dynamics.

DZ-2384 is an optimized derivative of a preclinical compound called AB-5, which, in turn, is a synthetic analogue of the natural product diazonamide A, which was originally isolated from the marine sponge Diazona angulata. In mouse models of pancreatic, colon, metastatic breast cancer and acute lymphocytic leukaemia, DZ-2384 was well tolerated and showed potent single-agent anticancer activity. Furthermore, in a xenograft model of human pancreatic ductal adenocarcinoma (PDAC), the effect of DZ-2384 treatment was synergistic in combination with gemcitabine, which is the current standard of care for PDAC. In a genetic model of PDAC, treatment with a combination of DZ-2384 and gemcitabine doubled response rates compared with gemcitabine alone. Potential neurotoxicity was investigated in rat models, and no electrophysiological or microscopic evidence for neuropathy was found at effective levels of the drug.

The authors then used RNA interference (RNAi) screens, surface plasmon resonance, X-ray crystallography and electron microscopy to show that the drug binds to the classic vinca alkaloid site at the interface between $\alpha$ - and $\beta$-tubulin, in exactly the same pocket as the MTA vinblastine. However, compared with vinblastine, which induces curvature in protofilaments and subsequent catastrophe, DZ-2384 was found to have a straightening effect on protofilaments. This interference with microtubule dynamics leads to apoptosis of dividing cells but, compared with vinblastine, it also increases the probability of rescue events in non-dividing cells.

These results demonstrate that a classic MTA-binding site on tubulin can be targeted in a different way, which results in a potent but less toxic candidate MTA for chemotherapy, and show that DZ-2384 is a promising candidate for the treatment of PDAC and a wide range of other cancers.

Alexandra Flemming

ORIGINAL ARTICLE Wieczorek, M. et al. The synthetic diazonamide DZ-2384 has distinct effects on microtubule curvature and dynamics without neurotoxicity. Sci. Transl Med. 8, 365ra159 (2016) 